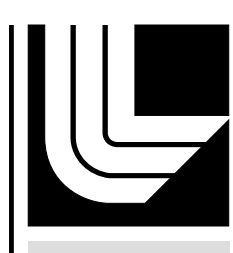

LAWRENCE LIVERM ORE NATIONAL LABORATORY

Modeling fast neutron irradiation damage accumulation in tungsten

J. Marian, T. Hoang

August 20, 2012

Journal of Nuclear Materials 
This document was prepared as an account of work sponsored by an agency of the United States government. Neither the United States government nor Lawrence Livermore National Security, LLC, nor any of their employees makes any warranty, expressed or implied, or assumes any legal liability or responsibility for the accuracy, completeness, or usefulness of any information, apparatus, product, or process disclosed, or represents that its use would not infringe privately owned rights. Reference herein to any specific commercial product, process, or service by trade name, trademark, manufacturer, or otherwise does not necessarily constitute or imply its endorsement, recommendation, or favoring by the United States government or Lawrence Livermore National Security, LLC. The views and opinions of authors expressed herein do not necessarily state or reflect those of the United States government or Lawrence Livermore National Security, LLC, and shall not be used for advertising or product endorsement purposes. 


\title{
Modeling fast neutron irradiation damage accumulation in tungsten
}

\author{
Jaime Marian and Tuan L. Hoang \\ Lawrence Livermore National Laboratory, Livermore, CA 94551
}

\begin{abstract}
Due to its advantageous physical properties, tungsten $(\mathrm{W})$ is being considered as a candidate structural material in fusion applications. In this paper, we perform stochastic cluster dynamics calculations of irradiation damage accumulation in pure $\mathrm{W}$ under fast neutron spectra in up to doses of $1.5 \mathrm{dpa}$ in the $400 \sim 600^{\circ} \mathrm{C}$ interval. Our calculations suggest that He bubbles and dislocation loops accumulate under fusion conditions, but not under fast fission spectra. We study the temperature dependence of swelling and find that it is maximum in the $550 \sim 590^{\circ} \mathrm{C}$ temperature range, falling precipitously above $600^{\circ} \mathrm{C}$. Swelling levels are very low, never surpassing a fraction of a percentage point. We also provide hardening estimates based on the accumulation of sessile dislocation loops under fusion conditions and show that they are moderate, ranging between 70 and $137 \mathrm{MPa}$ at $400^{\circ} \mathrm{C}$.
\end{abstract}

Keywords: tungsten, fusion materials, neutron irradiation

\section{Introduction}

Tungsten $(\mathrm{W})$ is being considered as a leading candidate for plasma-facing applications in magnetic fusion energy (MFE) devices. The most attractive properties of $\mathrm{W}$ for MFE are its high melting point and thermal conductivity, low sputtering yield and low long-term disposal radioactive footprint. These advantages are accompanied unfortunately with very low fracture toughness characterized by brittle trans- and inter-granular failure, which severely restrict the useful operating temperature window [1].

$\mathrm{W}$ is typically alloyed with $5 \sim 26$ at. $\%$ Re to increase low temperature ductility and improve high temperature strength and plasticity [2]. However, Re is an undesired impurity in MFE neutron environments due to its high transmutation rate into Os [3] and the possible formation of brittle $\sigma$ phases [4]. Even in pure $\mathrm{W}, \mathrm{Re}$ is the main transmutation product under fast neutron conditions and so understanding the irradiation behavior of the $\mathrm{W}$-Re system is paramount to component performance predictions.

Indeed, irradiations in EBR-II up to 9 dpa have revealed the absence of swelling in W-25\%Re [5] and W-10\%Re [6] compared to values of close to $2 \%$ in pure W. Matolich measured the temperature at which peak swelling occurs in pure $\mathrm{W}$ at approximately $750^{\circ}$ [5]. In a series of recent papers, Hasegawa and collaborators have shown that Re precipitation suppresses void and dislocation loop density accumulation and size growth $[7,8,9]$, although they observe that subsaturated Re precipitation above 1 dpa gives rise to very dramatic hardening increases. This precipitation of soluble Re under irradiation was observed by Williams et al. during EBR-II experiments in 1983 [10]. By comparison, the effect of transmutant He on $\mathrm{W}$ under fast neutron irradiation has been less studied ${ }^{1}$. Gilbert and Sublet [3] report relatively low He transmutation rates under ITER first-wall conditions ( $\approx 1$ appm per dpa). However, Kornelsen [11, 12] studied He accumulation in pure $\mathrm{W}$ crystals previously irradiated with heavy ions and observed the formation of He bubbles.

Computer simulations can provide insight into the fundamental mechanisms of damage accumulation and associated detrimental effects. At the atomistic scale, the treatment of W-Re alloys is limited to a few hundred atoms using electronic structure calculations due to the absence of suitable empirical 
potentials. By contrast, W-He potentials have been developed and used to assess the effect of He on Frenkel pair production in displacement cascades [13]. In addition, ion implantation studies in W have been published recently using energetics from electronic structure calculations [14]. However, to our knowledge, no damage accumulation simulations under fast neutron conditions in W exist. In this work we conduct simulations of void swelling and hardening in pure W including the effect of transmutant He in the temperature range of fusion materials. We analyze the nature of the vacancy and self-interstitial atom (SIA) type defects and discuss the implications for material degradation. We consider two distinct neutron environments. The first is that found in the fast-fission JOYO reactor, following the irradiations by Hasegawa et al. [9]. The second corresponds to ITER fusion neutron conditions, such as those considered by Gilbert and Sublet [3].

\section{The stochastic cluster dynamics method}

Here we use the stochastic cluster dynamics method (SCD) [15] to perform all simulations. SCD is a stochastic variant of the mean-field rate theory technique, alternative to the standard ODE-based implementations, that eliminates the need to solve exceedingly large sets of ODEs and relies instead on sparse stochastic sampling from the underlying kinetic Master Equation. Rather than dealing with continuously varying defect concentrations in an infinite volume, SCD evolves an integer-valued defect population $N_{i}$ in a finite material volume $V$, thus avoiding combinatorial explosion in the number of ODEs. This makes SCD ideal to treat problems where the dimensionality of the cluster size space is high, e.g., when multispecies simulations - for example involving heavy particles, $\mathrm{He}, \mathrm{H}$, etc., simultaneously- are of interest. SCD recasts the standard ODE system into stochastic equations of the form:

$$
\frac{d N_{i}}{d t}=\tilde{g}_{i}-\sum_{i} \tilde{s}_{i j} N_{i}+\sum_{j} \tilde{s}_{j i} N_{j}-\sum_{i, j} \tilde{k}_{i j} N_{i} N_{j}+\sum_{j, k} \tilde{k}_{j k} N_{j} N_{k}
$$

where the set $\{\tilde{g}, \tilde{s}, \tilde{k}\}$ represents the reaction rates of $0^{\text {th }}$ (insertion), $1^{\text {st }}$ (thermal dissociation, annihilation at sinks), and $2^{\text {nd }}$ (binary reactions) order kinetic processes taking place inside $V$.

In this work we use $V=10^{-19} \mathrm{~m}^{3}$ in all the simulations and ensure at all times that $V^{\frac{1}{3}}>\ell$, where $\ell$ is the maximum diffusion length $l_{i}$ of any species $i$ in the system, defined as:

$$
\begin{gathered}
\ell=\max _{i}\left\{l_{i}\right\} \\
l_{i}=\sqrt{\frac{D_{i}}{R_{i}}}
\end{gathered}
$$

Here, $D_{i}$ and $R_{i}^{-1}$ are the diffusivity and the lifetime of a mobile cluster within $V$. From eq. (1), $R_{i}=$ $\tilde{s}+\sum_{j} \tilde{k}_{i j} N_{i}$. In other words, we ensure that the characteristic diffusion length of any defect species is contained within the simulation volume at any given time.

\section{Method parameterization}

\subsection{Neutron energy spectra}

Although Hasegawa et al. do not specify the reactor location where their samples were irradiated, here we take the flux at the reflector region as an upper bound of that used for materials testing in more outer locations [16]. For its part, the ITER flux corresponds to the equatorial plane of the first wall toroidal structure [3]. Both neutron spectra are given in Fig. 1 for comparison. 


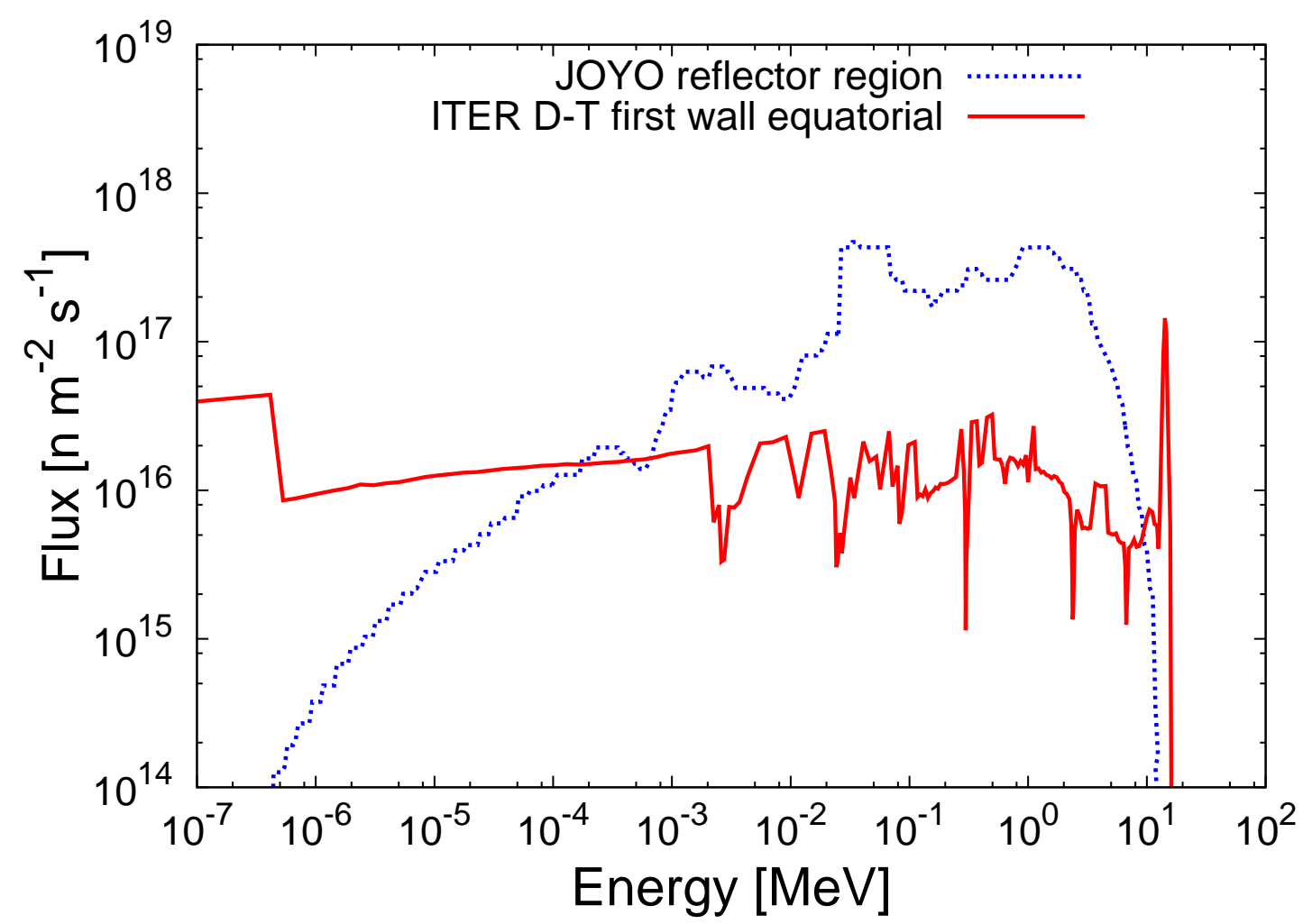

Figure 1: Neutron flux spectra for the two locations considered in this work. The peak at $\approx 14 \mathrm{MeV}$ for the ITER flux can be clearly appreciated.

\subsection{Source term determination}

SCD is ideally suited to deal with the probabilistic aspects of irradiation damage. Once a suitable source function is constructed, one can accurately sample it to create defect distributions. Establishing the source term requires the calculation of recoil energy distributions and gas production for the neutron spectra given above. These are generally very difficult to obtain experimentally [17] and those used here have been obtained using the SPECTER code [18] for both the JOYO and ITER neutron fluxes. The spectral-averaged recoil energy distributions are shown in Fig. 2, while the He production is given in Table 1 . The recoil spectra are given as cumulative distribution functions, $C\left(E_{P K A}\right)$. These functions are used to obtain random samples of the primary knock-on atom (PKA) energies $E_{P K A}$ by solving $E_{P K A}=$ $C^{-1}(\xi)$, where $\xi$ is a random number uniformly distributed in $[0,1)$. Note that $C(E)=\int_{0}^{E} P(E) d E$, where $P(E)$ is the normalized recoil energy spectrum.

The production of He in the selected location at JOYO can be considered negligible, consistent with a threshold of $0.3 \mathrm{MeV}$ for the ${ }^{184} \mathrm{~W}(\mathrm{n}, \alpha)^{181} \mathrm{Hf}$ channel [19]. As we shall show, this has a large effect on the ensuing microstructural evolution under irradiation.

$C\left(E_{P K A}\right)$ gives the cumulative PKA probability as a function of their energy $E_{P K A}$. However, the complete characterization of the damage source term requires that these PKA energies be expressed in terms of the number and classification of the point defects produced. This has been the subject of very recent molecular dynamics studies in pure $\mathrm{W}[22,20,21]$, and, on the basis of these studies, we have taken a damage production of law of the form:

$$
N=a E_{P K A^{\prime}}^{b}
$$

where $N$ is the number of Frenkel pairs produced. Here $a$ and $b$ are adjustable parameters that take 


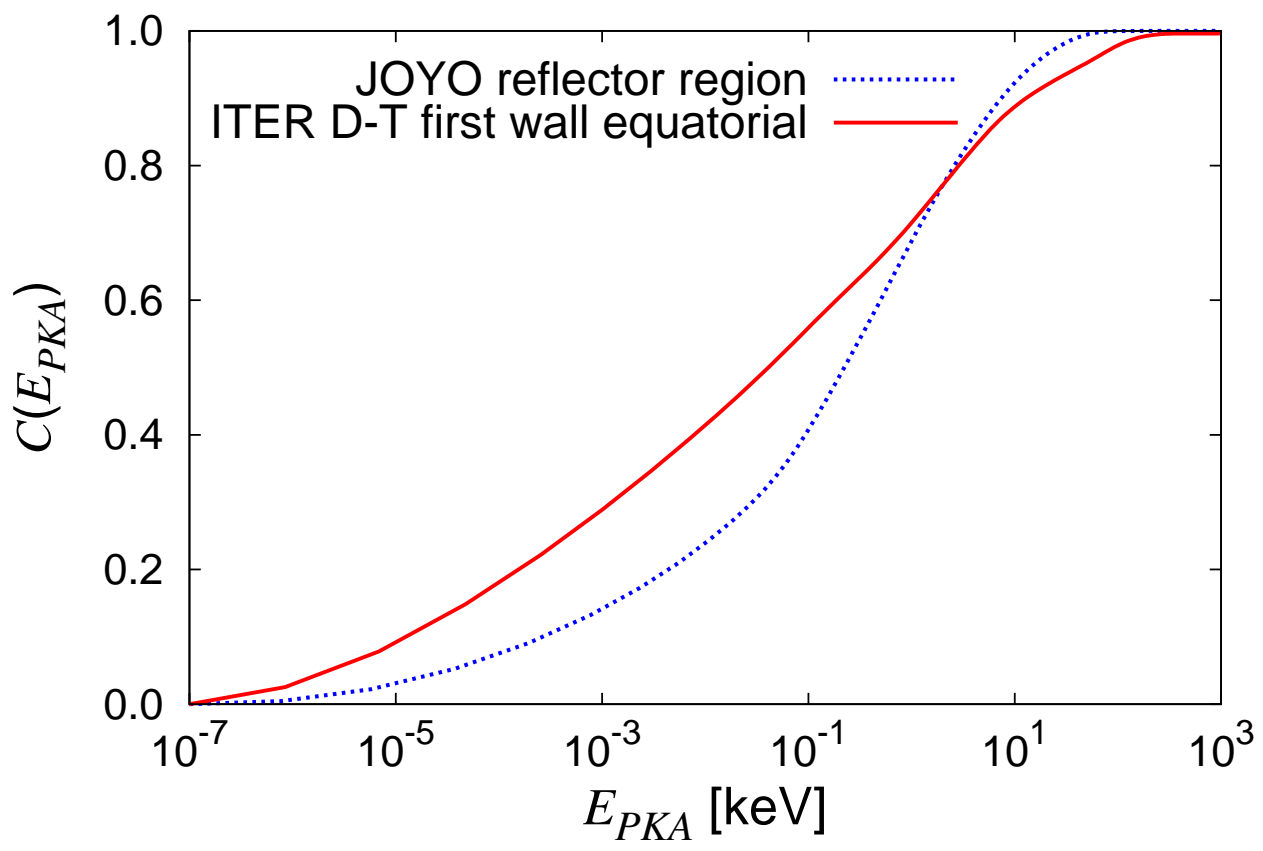

Figure 2: Recoil cumulative distribution function $C\left(E_{P K A}\right)$ in $\mathrm{W}$ corresponding to the neutron spectra given in Fig. 1.

values of 1.49 and 0.82, respectively, when $E_{P K A}$ is expressed in keV. After Fikar and Schäublin [22], we assume that the fraction of SIAs and vacancies in clusters is, on average, 0.5 and 0.2. In accordance with these authors, we consider a cluster to be formed when two or more defects aggregate together.

\subsection{System energetics}

The calculations described here involve three distinct species, namely, SIAs, vacancies and He atoms, and any kind of reaction among them and their clusters is allowed. The energetics to enable these reactions have been obtained by Becquart and Domain using electronic structure calculations and published in a series of recent papers [23, 24]. In these works, migration parameters for all single-species objects are provided regardless of size-i.e. all pure vacancy, SIA, and He clusters are considered mobile- which is the approach used here as well. Conversely, He bubbles are sessile, while their thermal stability is dictated by the following relations for the dissociation energies of He atoms and vacancies from V-He clusters:

$$
E_{d}^{V}=2.4+3.5 \log x+1.7 \log ^{2} x,
$$

and

$$
E_{d}^{H e}=4.6-1.1 \log x-0.3 \log ^{2} x,
$$

where $x$ is the He-to-vacancy ratio in the cluster. The functional form of these expressions has been taken following the work by Terentyev et al. [25], and the fitting data from Ref. [24].

In addition, we have enabled SIA and SIA cluster reactions with He atoms in the same manner as done in our previous study in Fe [15], i.e. any collision between He and SIA clusters results in a SIA-He sessile structure that is thermally stable. In fact, this becomes the sole mechanisms of SIA loop accumulation in our calculations, as we shall see. Similarly, He bubbles are considered immobile (albeit their . The entrapment of He by lattice defects of both vacancy and SIA type in W was confirmed by works such as that of Kornelsen more than four decades ago [11, 12]. More details about the energetics employed here can be found in the above cited references. 
Table 1: Irradiation parameters and description of simulations performed.

\begin{tabular}{|c|c|c|c|}
\hline & \multicolumn{3}{|c|}{ JOYO irradiations } \\
\hline Dose rate $\left(\mathrm{dpa}^{-1}\right)$ & \multicolumn{3}{|c|}{$3.5 \times 10^{-7}$} \\
\hline He production (appm dpa ${ }^{-1}$ ) & \multicolumn{3}{|c|}{$2.5 \times 10^{-11}$} \\
\hline Temperature $\left({ }^{\circ} \mathrm{C}\right)$ & 400 & 538 & 583 \\
\hline Dose (dpa) & 0.17 & 0.40 & 0.96 \\
\hline & \multicolumn{3}{|c|}{ ITER irradiations } \\
\hline Dose rate $\left(\mathrm{dpa} \mathrm{s}^{-1}\right)$ & \multirow{2}{*}{\multicolumn{3}{|c|}{$\begin{array}{c}5.8 \times 10^{-8} \\
1.1\end{array}$}} \\
\hline He production (appm dpa $\left.{ }^{-1}\right)$ & & & \\
\hline Temperature $\left({ }^{\circ} \mathrm{C}\right)$ & 400 & 500 & 550 \\
\hline Dose (dpa) & 0.10 & 0.44 & 1.54 \\
\hline Hardening $(\mathrm{MPa})$ & $68.5 \sim 136.9$ & $23.1 \sim 46.2$ & $8.4 \sim 16.8$ \\
\hline
\end{tabular}

\subsection{Microstructural defect sinks}

Dislocations and grain boundaries are considered inexhaustible defect sinks in our simulations. We assume that the irradiated material corresponds to well-annealed polycrystalline $\mathrm{W}$ with a dislocation density of $\rho_{d}=10^{14} \mathrm{~m}^{-2}$ and an average grain size of 100 microns [26]. Dislocations are assumed to absorb SIA and SIA clusters preferentially over vacancies with a bias of $10 \%$.

\section{Results}

\subsection{Swelling as a function of temperature}

The energetics at hand dictate that vacancy mobility in $\mathrm{W}$ is relatively low in the temperature range explored here. This allows small $\mathrm{V}$ clusters, either generated directly in displacement cascades or formed upon the subsequent time evolution, to accumulate at first. In the absence of stabilizing He atoms, the long-term fate of these clusters, especially those containing five or less vacancies, is questionable. In addition, fast-diffusing SIAs and small SIA clusters probe the configurational space in 3D, leading to recombination and mutual annihilation.

However, a critical population of clusters does survive and grows to become thermally stable. This is a temperature-dependent process, set by the timescale. The accumulated tally of these (stable) V clusters gives the total swelling of the material ${ }^{2}$. The percentage swelling in the 400 -to- $600{ }^{\circ} \mathrm{C}$ range for both the JOYO and ITER scenarios is plotted in Fig. 3. The labels assigned to each data point indicate the dose at which the swelling tally was obtained. Owing to their relatively high mobility, single vacancies are not included in the swelling calculation. Similarly, di-vacancies are not considered stable due to their negative binding energy and are also not tallied unless they are found in the form of $2 \mathrm{~V}-n \mathrm{He}$ complexes for any $n>0$. Calculations performed at and above $600^{\circ} \mathrm{C}$ show negligible swelling in both cases. As the figure shows, the ITER environment results in approximately $30 \%$ higher swelling than JOYO at $400^{\circ} \mathrm{C}$. However, the values converge as the temperature increases.

By itself, the information plotted in Fig. 3 does not expose the differences in temperature and dose in full detail. For that, it is useful to look at the size partition of the void subpopulation. Fig. 4 shows a composite histogram including all cases simulated here. For size calculations, it is assumed that vacancy clusters adopt spherical shapes and SIA loops are arranged as circular loops. 'Size' refers to their respective diameters. Here the distinctions are clearer. In both the JOYO and ITER cases, a clear shift in the size distribution is observed as a function of temperature. For example, at $400^{\circ} \mathrm{C}$-for which the swelling is highest - voids appear mostly in the form of small clusters less than $2 \mathrm{~nm}$ in diameter. The average void size at the higher temperatures, on the contrary, shifts to approximately 3 and $5 \mathrm{~nm}$, with no voids observed below $1.5 \mathrm{~nm}$. With respect to neutron spectrum effects, both the number and average void size for 


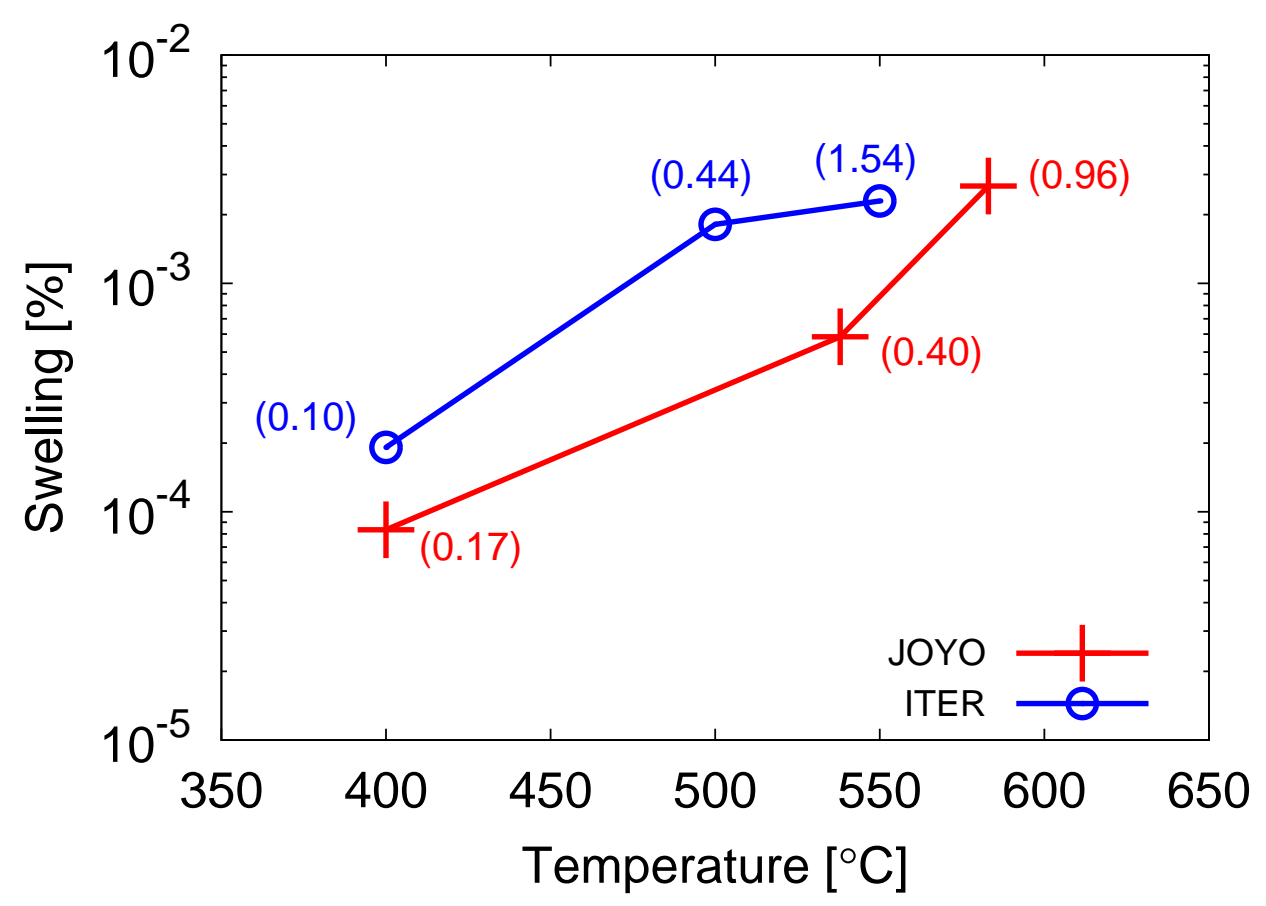

Figure 3: Temperature dependence of swelling in pure W under JOYO and ITER irradiation conditions. The numbers in parentheses correspond to the accumulated dose expressed in dpa for each case.

each temperature are higher for the ITER irradiations. A first-order explanation for this behavior resides in the role of He (again, present in ITER, absent in JOYO). Superposed with the total vacancy cluster size distribution in Fig. 4, we have plotted the fraction of clusters containing He atoms. In view of the results, we can conclude that He has two main effects, namely, to increase the population of total clusters (likely by the enhanced production of stable void nucleii), and to play an increasingly more important role the higher the temperature (all the voids at $550^{\circ} \mathrm{C}$ contained He atoms). The buildup of He bubbles in ITER is shown in Fig. 5.

Another important effect induced by He-atom production is the accumulation of SIA loops. Evidently, no density of loops builds up in the JOYO scenario. By contrast, in the ITER case, a gradual buildup occurs at $400^{\circ} \mathrm{C}$, while at 500 and $550^{\circ} \mathrm{C}$ saturation tendencies can be appreciated after 0.1 and $0.03 \mathrm{dpa}$, respectively. Whether this will be the eventual outcome also at $400^{\circ} \mathrm{C}$ is unclear at this point. What is clear is the strong inverse temperature dependence of SIA loop accumulation, also in agreement with the established understanding about irradiation hardening in bcc metals. As for swelling, no appreciable accumulation of SIA loops occurred above the temperatures studied here.

Once more, it is informative to plot the SIA loop subpopulation in terms of the size of the equivalent circular disc corresponding to the number of constituent defects. This is done in Fig. 6.

\subsection{SIA loop hardening}

The equivalent integrated quantity associated to the SIA loop size histogram is hardening, expressed in our context as:

$$
\Delta \sigma=M \alpha \frac{\mu b}{4 \pi l}
$$




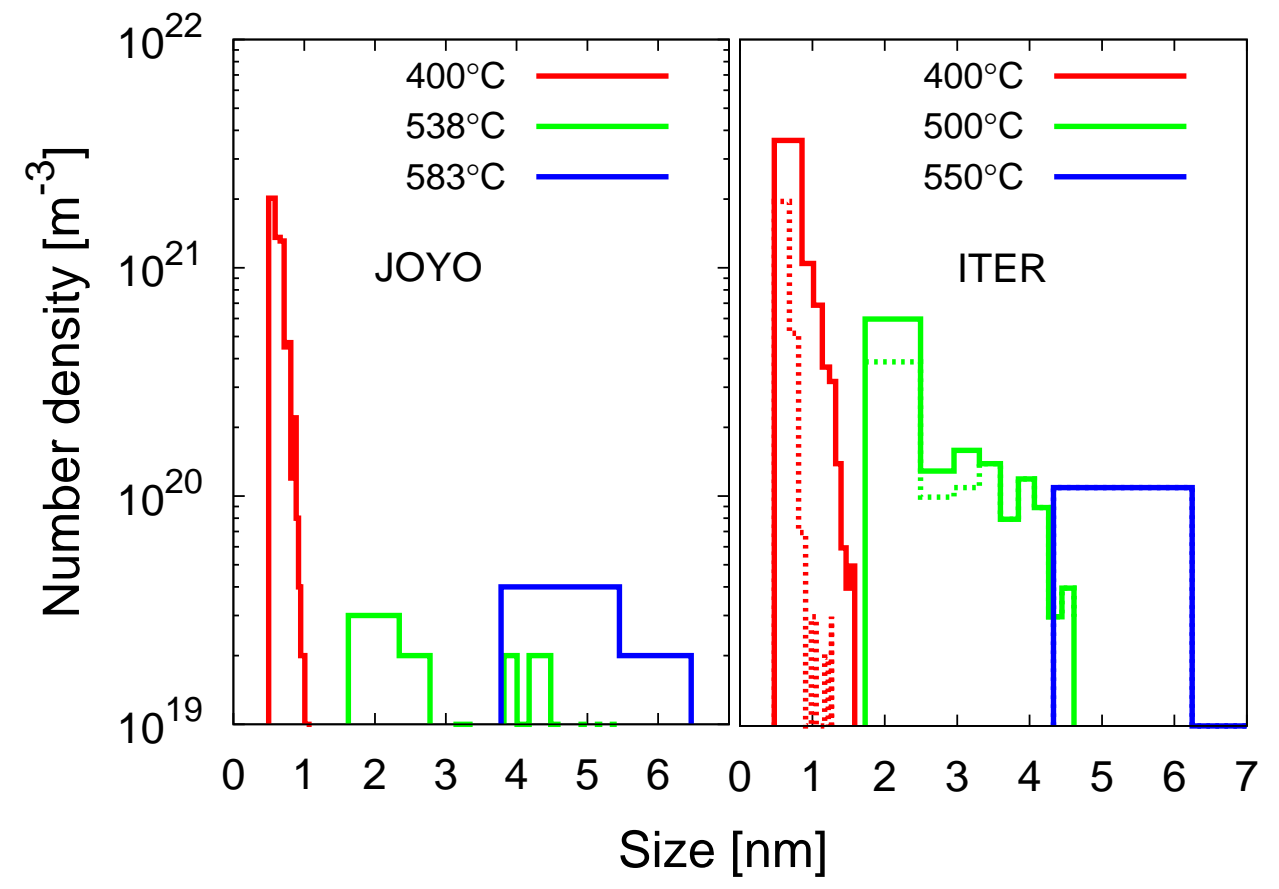

Figure 4: Vacancy cluster size histogram at the point of maximum dose (cf. Fig. 3) for JOYO and ITER irradiations. The dashed line in the ITER case represents the fraction of voids containing He.

where $M$ is the Taylor factor, $\alpha$ is a strength coefficient, $\mu$ the shear modulus, $b$ is the Burgers vector, and $l$ the distance between obstacles. $l$ can be computed as $\left(\bar{D} \rho_{t}\right)^{-\frac{1}{2}}$, where:

$$
\bar{D} \rho_{t}=\int_{V} D(n) \rho(n) d n,
$$

is the product of the effective loop diameter $\bar{D}$ and the total loop density $\rho_{t}$. Here $D(n)$ is the diameter of a loop of $n$ SIAs and $\rho(n)$ the corresponding number density. The integral can be approximated by a sum of discrete bins describing the histogram of loop sizes such as that shown in Fig. 6 . For values of $M=2.75$ (for bcc crystals) [27], and $\alpha=0.25 \sim 0.50$ we obtain increases in yield stress that range between $137 \mathrm{MPa}$ at $600^{\circ} \mathrm{C}$ and $8 \mathrm{MPa}$ at $550^{\circ} \mathrm{C}$. The actual range of $\Delta \sigma$ values for each temperature is given in Table 1.

It is worth mentioning that voids and He bubbles are likely to have their own non-negligible contribution to hardening. However, having multiple strengthening mechanisms introduces a new set of complications, particularly associated with which rule of mixtures to use. We get back to this issue in the next section.

\section{Discussion and conclusions}

We have carried out SCD simulations of fast neutron damage accumulation in pure $\mathrm{W}$ in the temperature range of interest to fusion. We have computed swelling and hardening and have analyzed the size distributions of vacancy and SIA clusters. The simulations have been conducted up to doses of the order of those reached by Hasegawa et al. at each temperature in experiments in JOYO [9].

We have considered spectral effects by studying two fast neutron irradiation scenarios. JOYO corresponds to a fast fission reactor with limited neutron yields above $1 \mathrm{MeV}$. By contrast, the ITER fusion flux consists of mainly 14-MeV neutrons combined with a long energy scattering tail. The main difference for 


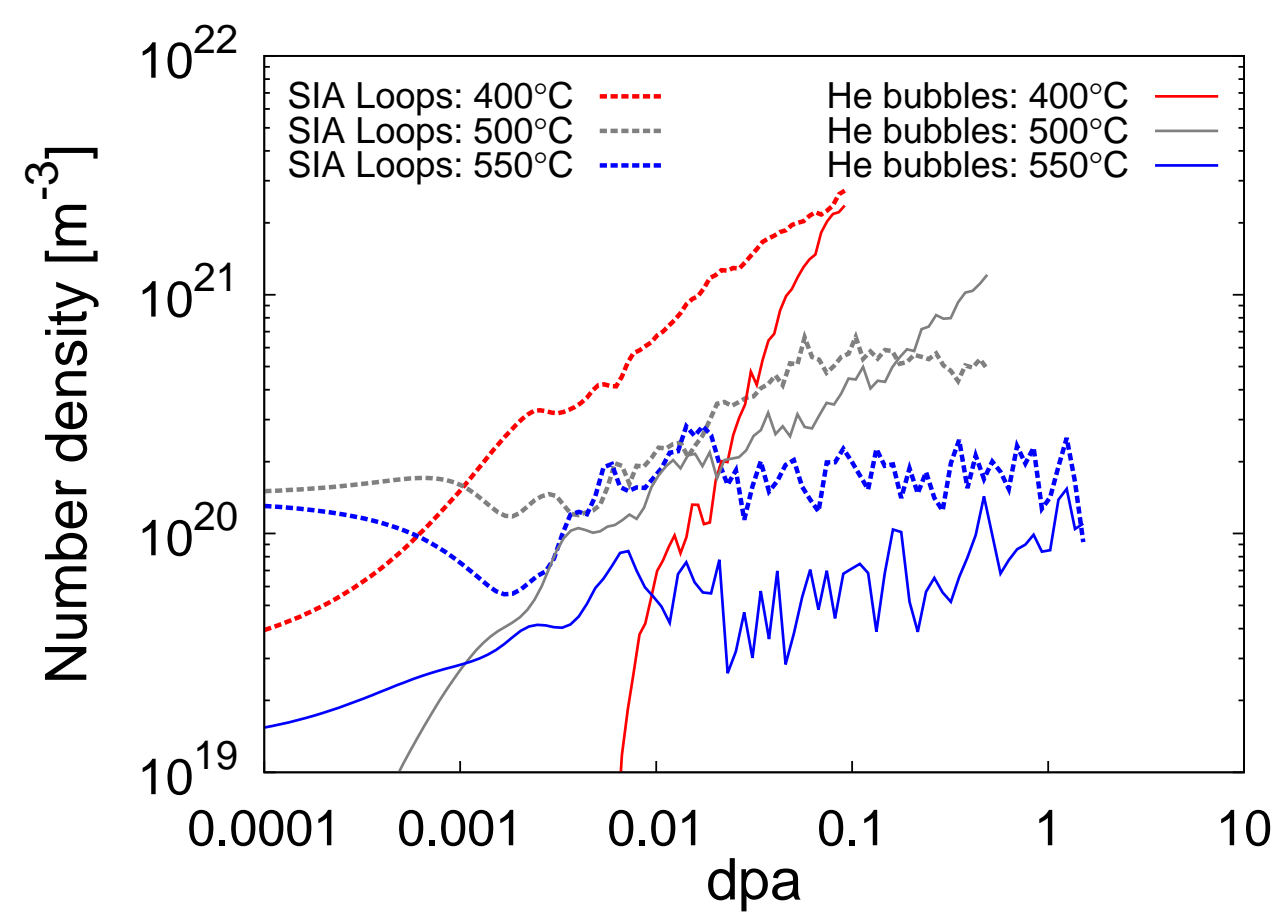

Figure 5: Accumulation of visible $(>1.0 \mathrm{~nm})$ SIA loops and He bubbles as a function of dose and temperature for the ITER irradiations.

our purposes is the amount of transmutant He produced in each case. He is found in two thirds of the voids formed under ITER conditions, with incubation times for He bubble formation that are inversely proportional to temperature and range from $7.5 \times 10^{-5} \mathrm{dpa}$ at $550^{\circ} \mathrm{C}$ to $0.007 \mathrm{dpa}$ at $400^{\circ} \mathrm{C}$, cf. Fig. 5 .

The effect of temperature on defect size and number density can be clearly appreciated in Figs. 4 and 6. Void size becomes increasingly larger and the void distribution increasingly more homogeneous at the highest temperature simulated here. In contrast, SIA loop size and density are highest at $400^{\circ} \mathrm{C}$ and gradually decrease with temperature.

However, at $<0.01 \%$, our calculations yield swelling levels in pure $\mathrm{W}$ that are very low, of the order of three orders of magnitude lower than those measured experimentally in similar conditions [5]. Peak swelling in our calculations is attained in the $550 \sim 600^{\circ} \mathrm{C}$ interval, below experimental measurements of around $800^{\circ} \mathrm{C}$. Moreover, in our calculations we find that swelling and hardening abruptly decrease after $600^{\circ} \mathrm{C}$, irrespective of conditions. Regarding hardening, estimates obtained from a simple disperse barrier model yield values that are also lower than those measured in real samples irradiated to comparable levels and temperatures.

Evidently, there are some key physical processes that impact microstructural evolution that are missing in our simulations:

- The presence of transmutants and the consideration of reaction induced precipitation, particularly of Re and Os, has profound implications on the microstructural evolution of $\mathrm{W}$ under irradiation. Our models at present do not capture the effect of these impurities and alloy elements

- Recent fast neutron irradiations of pure W reveal the formation of void lattices at and above approximately $1 \mathrm{dpa}$ [28]. Void lattices are evidence of the 1D nature of SIA cluster diffusion [29, 30], which is not captured in our simulations.

- He plays a very important role in our calculations, enhancing void swelling and enabling SIA loop 


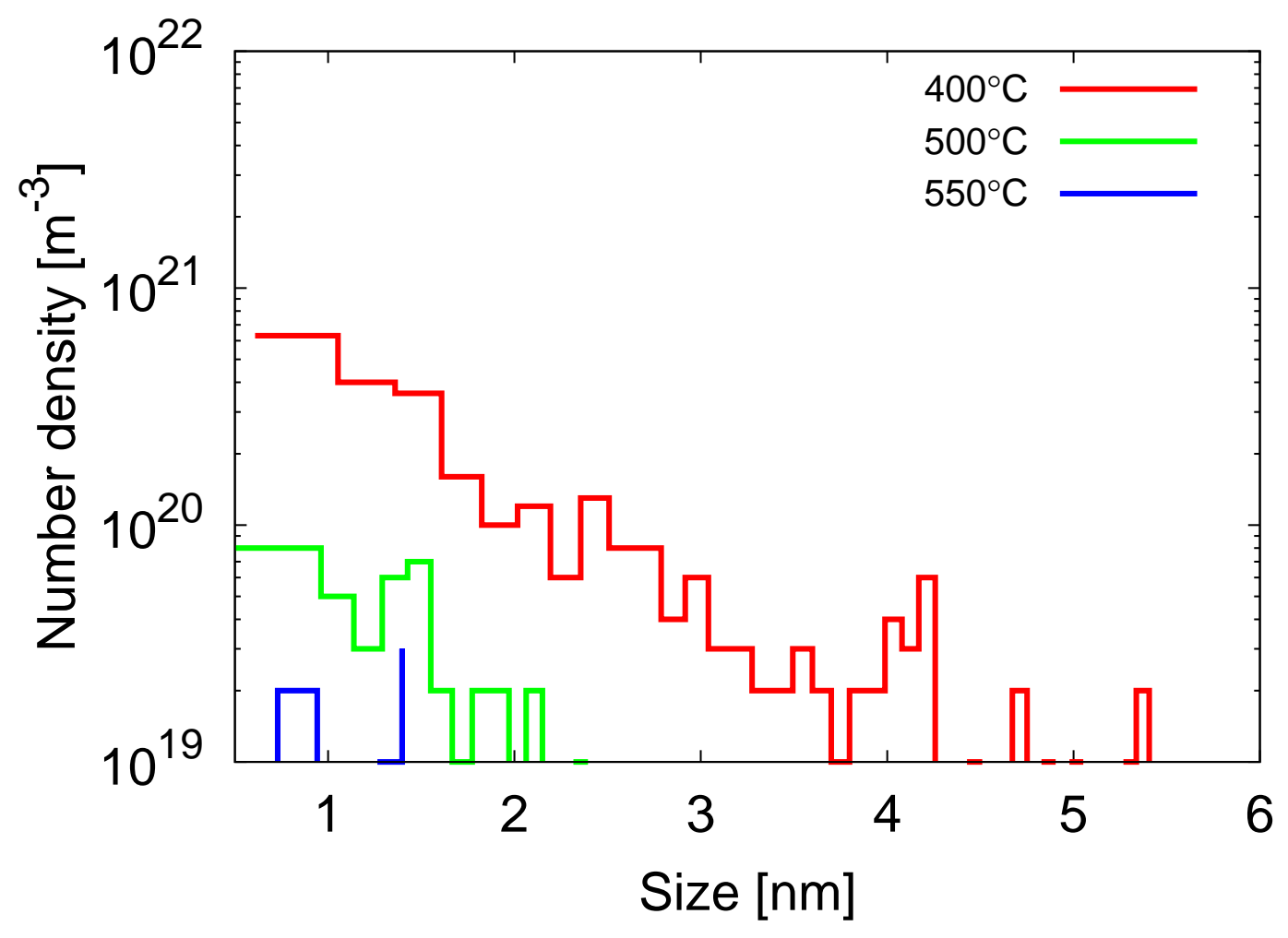

Figure 6: SIA cluster size histogram at the point of maximum dose (cf. Fig. 3) for the ITER irradiations.

accumulation. It is not clear whether its effect is exaggerated in our simulations compared to realistic scenarios.

- Voids and He bubbles are sure to contribute to hardening in addition to loop hardening. Void hardening can be approximated by using models such as that of Scattergood and Bacon [31]. However, we have not included it here to isolate the effect due to SIA loops and avoid issues with the rule of mixtures when more than one strengthening mechanism is included.

Simulations of ion implantation do not suffer from this limitation, as transmutation elements are of no concern. Even then, one of the main conclusions of the most visible ion implantation simulation study is that close linkage with experiments is needed to advance the fidelity of computer models [14].

In any case, we have demonstrated the connection between damage accumulation kinetics and observable engineering variables such as swelling and hardening in pure W. Future work will be devoted to mitigating some of the limitations pointed out above.

\section{Acknowledgments}

This work was performed under the auspices of the U.S. Department of Energy by Lawrence Livermore National Laboratory under Contract DE-AC52-07NA27344. We acknowledge support from the Laboratory Directed Research and Development Program under project 11-ERD-023.

\section{Notes}


${ }^{1}$ Not so for low-energy He deposition on W surfaces, as is relevant for plasma-wall interaction in MFE reactors, where a voluminous literature exists.

${ }^{2} \mathrm{As}$ is customary in the rate theory literature, this is assuming that the vacancy relaxation volume is equal to the atomic volume, and that the swelling contribution of a $n \mathrm{~V}$ cluster is $n \Omega_{a}$, where $\Omega_{a}=15.9 \AA^{3}$ is the atomic volume.

\section{References}

[1] S. J. Zinkle and N. M. Ghoniem, Journal of Nuclear Materials 417 (2011) 2.

[2] E. Lassner and W. F. Schubert, Tungsten: Properties, Chemistry, Technology of the Element, Alloys, and Chemical Compounds (Kluwer Academic/Plenum Publishers, New York, NY, 1999).

[3] M. R. Gilbert and J. C. Sublet, Nucl. Fusion 51 (2011) 043005.

[4] G. A. Cottrell, J. Nucl. Mater. 334 (2004) 166.

[5] J. Matolich, H. Nahm and J. Moteff, Scripta Metall. 8 (1974) 837

[6] R. Herschitz and D. N. Seidman, Acta Metall. 32 (1984) 1141.

[7] J. C. He, A. Hasegawa, and K. Abe, J. Nucl. Mater. 377 (2008) 348.

[8] T. Tanno, A. Hasegawa, J. C. He, M. Fujiwara, M. Satou, S. Nogami, K. Abe and T. Shishido, J. Nucl. Mater. $386-388$ (2009) 218

[9] A. Hasegawa, T. Tanno, S. Nogami and M. Satou, J. Nucl. Mater. 417 (2011) 491.

[10] R. K. Williams, F. W. Wiffen, J. Bentley and J. O. Stiegler, Metall. Trans. A 14A (1983) 655.

[11] E. V. Kornelsen, Canadian Journal of Physics 48 (1970) 2812.

[12] E. V. Kornelsen, Radiation Effects 13 (1972) 227.

[13] N. Juslin, V. Jansson and K. Nordlund, Phil. Mag. 90 (2010) 3581.

[14] C. S. Becquart, M. F. Barthe, and A. De Backer, Phys. Scr. T145 (2011) 014048.

[15] J. Marian and V. V. Bulatov, J. Nucl. Mater. 415 (2011) 84.

[16] http://www.jaea.go.jp/04/o-arai/joyo_users_guide/joyomk3/spectrum/main.html

[17] D. N. Seidman, R. S. Averback and K. Benedek, Physica Satus Solidi (b) 144 (1987) 85.

[18] SPECTER: Neutron Damage Calculations for Materials Irradiations, by L. R. Greenwood and R. K. Smither, ANK/FPP/TM-197.

[19] http://www.nndc.bnl.gov/exfor/endf02.jsp

[20] J. Fikar and R. Schäublin, J. Nucl. Mater. 386-388 (2009) 97.

[21] T. Troev, N. Nankov and T. Yoshiie, Nucl. Inst. Meth. Phys. Res. B 269 (2011) 566.

[22] J. Fikar and R. Schäublin, Nucl. Inst. Meth. Phys. Res. B 255 (2007) 27.

[23] C. S. Becquart and C. Domain, J. Nucl. Mater. 385 (2009) 223.

[24] C. S. Becquart, C. Domain, U. Sarkar, A. DeBacker and M. Hou, J. Nucl. Mater. 403 (2010) 75.

[25] D. Terentyev, N. Juslin, K. Nordlund, and N. Sandberg, Journal of Applied Physics 105 (2009) 103509.

[26] W. Knauss (Plansee Corp.), private communication.

[27] Yu. M. Platov, V. M. Lazorenko,, V. I. Tovtin and F. A. Khasanov, Inorganic Materials: Applied Research 2 (2011) 37.

[28] A. Hasegawa, presented at the $15^{\text {th }}$ International Conference on Fusion Reactor Materials, Charleston, SC, October 16-22, 2011.

[29] H. L. Heinisch and B. N. Singh, Phil. Mag. 83 (2003) 3661.

[30] J. H. Evans, Phil. Mag. 85 (2005) 1177.

[31] R. O. Scattergood and D. J. Bacon, Acta Metall. 30 (1982) 165. 